\title{
GeoDataHub: Building centralised repository of authoritative cartographic and geospatial resources
}

\author{
Igor Drecki \\ The University of Auckland, Auckland, New Zealand, i.drecki@auckland.ac.nz
}

Keywords: Cartographic Heritage, Centralised Repository, Online Resources

\begin{abstract}
:
GeoDataHub (geodatahub.library.auckland.ac.nz) is an authoritative national repository of cartographic and geospatial resources curated by the University of Auckland Library for research, government, and educational communities, as well as the public of New Zealand. Its objectives are to digitise cartographic materials, provide integrated and enhanced data access, and build a unique knowledge base concerning New Zealand's cartographic heritage. GeoDataHub showcases conceptual developments in cartography, surveying, photogrammetry, and other mapping, earth, and social science disciplines, highlighting their contribution to the development of the nation. It supports a dynamic, innovative, and leading edge historical and GIScience research, facilitating scientific discovery and knowledge building.
\end{abstract}

The repository contains digitised maps and charts published by authoritative agencies and research institutes, aerial photography provided by local authorities and all of government satellite imagery. A selection of key geospatial datasets produced by the government, Crown research institutes and local authorities augment the repository - currently over 30TB of data. The focus of GeoDataHub is on providing cartographic and geospatial resources for New Zealand, its offshore islands and territories; South Pacific, particularly Cook Islands, Niue and Tokelau; and Ross Dependency in Antarctica. Regional coverage primarily includes Auckland and Waikato, and parts of Northland, Bay of Plenty and East Cape. Local datasets cover primarily Auckland Region but a selection of datasets from other regions is also included. GeoDataHub is augmented by physical collection of maps and charts, atlases, aerial photographs, gazetteers, map indexes and catalogues, and cartographic paraphernalia, located in the Map Room of the University of Auckland Library.

This paper talks about challenges associated with the design, implementation and provision of content for GeoDataHub. Apart from building an integrated and enhanced data access application, facilitating textual, spatial and temporal search, metadata harvesting and discovery utilising GeoNetwork, a catalogue application that manages spatially referenced resources (www.geonetwork-opensource.org), the repository provides a further two ways of accessing cartographic and geospatial resources. One involves browsing the selected content of the repository, examining and downloading the material of interest; the other allows users to 'map a drive' and access all materials using familiar folder structure (the latter applies to the University community of users only). This approach however, occasionally requires provision of the same datasets in two different formats (compressed and uncompressed) depending how the users are accessing them. In addition, a knowledge base concerning New Zealand cartographic heritage is being implemented, providing a useful guide to authoritative mapping served by GeoDataHub. A particular challenge concerns documenting their provenance, content, extent and general characteristics in a modular fasion .

The paper concludes with presenting plans for extending GeoDataHub. This includes not only the creation of further geospatial records, provision of more content and expanding the knowledge base, but also strategic decisions concerning the selection of cartographic and geospatial resources and how to provide the best service possible to our growing user base. 